\title{
Optical Sensor of Anionic Surfactants Using Solid-Phase Extraction with a Lactone-form Rhodamine B Membrane
}

\author{
Takashi Masadome ${ }^{\dagger}$ and Motoo Akatsu \\ Department of Applied Chemistry, Faculty of Engineering, Shibaura Institute of Technology, \\ Toyosu, Koto, Tokyo 135-8548, Japan
}

\begin{abstract}
An optical sensor for the detection of anionic surfactants was developed. The optical sensing membrane is a 2-nitrophenyloctyl ether-plasticized poly(vinyl chloride) membrane incorporating a lactone-form Rhodamine B (L-RB). The response of the optical membrane to anionic surfactants was a result of the ion-pair coextraction of an anionic surfactant and a proton into the PVC membrane. The L-RB forms an ion associate with the extracted anionic surfactant; simultaneously, the formed L-RB ion associate is accompanied by a spectral change. Namely, the extracted anionic surfactant changes the color of the membrane from light pink to dark pink (absorption maximum; $558 \mathrm{~nm}$ ). The optical membrane responds to anionic surfactants, such as dodecylbenzenesulfonate, dodecylsulfate and di-2-ethylhexyl sulfosuccinate in the concentration range from 1 to $50 \mu \mathrm{M}$.
\end{abstract}

(Received February 5, 2008; Accepted April 28, 2008; Published June 10, 2008)

\section{Introduction}

The determination of anionic surfactants in aqueous solutions is very important in the process control of anionic surfactants and the monitoring of environmental water, and so on, because of their toxicity to various organisms. Solvent extractionspectrophotometric methods based on the formation of an ionpair of the anionic surfactant with a cationic dye, such as Methylene Blue, and extraction of the resulting ion-pair into a suitable organic solvent, such as chloroform, have been widely used for the determination of anionic surfactants. ${ }^{1-3}$ However, the procedures are very complicated and time-consuming. Furthermore, much poisonous organic solvent was often used in this method. A simpler method for the determination of anionic surfactants without using a poisonous organic solvent is required. The development of optical sensors for the determination of surfactants is expected because they have the advantages of being simple, safe and rapid. ${ }^{4-10}$ Hence, optical sensors have become very useful analytical tools. Several reports on optical sensors for the determination of surfactants have been published so far. An optical detection of cationic surfactants using an environment-sensitive fluorophore, 5-[(2aminoethyl)amino]naphthalene-1-sulfonate, has been reported by Seitz et al. ${ }^{11}$ The optical sensor responds to a cationic surfactant in the concentration range from 0.1 to $1 \mathrm{mM}\left(\mathrm{M}=\mathrm{mol} \mathrm{dm}^{-3}\right)$. A cationic surfactant biosensor reported by Lundgren et al. ${ }^{12}$ uses acrylodan-labeled bovine serum albumin as the biorecognition element. The biosensor responds to cationic surfactants in the concentration range from 5 to $60 \mu \mathrm{M}$. The sensor also responds to anionic surfactants. The detection range of the optical sensor to anionic surfactants is from 75 to $600 \mu \mathrm{M}$. The sensitivity of the optical sensor is not sufficient. The response characteristics of a $\mathrm{K}^{+}$ion-selective optrode to a cationic surfactant have been

$\dagger$ To whom correspondence should be addressed.

E-mail: masadome@sic.shibaura-it.ac.jp reported by Kawabata et al. ${ }^{13}$ The sensitivity of the optrode to the $\mathrm{K}^{+}$ion decreases with an increase in the concentration of the cationic surfactant. However, the response of the optrode to anionic surfactants has not been reported in detail. Wang et al. ${ }^{14}$ reported that polymeric films doped only with chromophores, such as $2^{\prime} 7^{\prime}$-dichlorofluorescein octadecyl ester or ETH 5350, were useful for the determination of nonionic surfactants, Triton $\mathrm{X}-100$ and Brij 35 , in the range of 5 to $300 \mu \mathrm{M}$ using $0.1-0.01$ $\mathrm{M}$ potassium or barium ions as background.

However, only a few reports on anionic surfactant optical sensor have been published so far. A plasticized poly(vinyl chloride) (PVC) membrane incorporating 1,2-benzo-7(diethylamino)-3-(octadecanoylimino)phenoxazine (ETH 5294) was used as an excellent reversible optical sensor for anionic surfactants. ${ }^{15}$ The response to anionic surfactants was the result of an ion-pair coextraction of the surfactant anion and a proton. When an optode membrane was exposed to an anionic surfactant solution, red ETH 5294, in its neutral form, was converted to a blue ETH 5294 in its protonated form, while inducing an absorption spectral change of the membrane. The sensitivity of the optical sensor is relatively high. The detection range of the optical sensor is from 20 to $1000 \mu \mathrm{M}$ for NaDBS. However, ETH 5294 is very expensive.

On the other hand, lactone-form Rhodamine B (L-RB) is often used as a suitable ion-association reagent, or a color reagent. ${ }^{16-21}$ The photometric determination of ethanol in liquors using L-RB as a color reagent has been reported by Yamamoto and Abe. ${ }^{16}$ The determination of anionic surfactants by flow injection analysis (FIA) coupled with liquid-liquid extraction using L-RB has been reported by Motomizu and Kobayashi. ${ }^{17}$ Rhodamine B (RB) can be readily extracted into benzene as its colorless L-RB. The L-RB reacts with an anionic surfactant to form an ion-association complex, which is extracted into benzene. This phenomenon was applied to the FIA of anionic surfactants in the concentration range from 5 to $50 \mu \mathrm{M}$.

L-RB can be easily immobilized in an organic membrane, such as a plasticized PVC membrane, because L-RB is soluble 
in an organic solvent, such as benzene. Furthermore, RB is very cheap and L-RB is easy to prepare. ${ }^{16,17}$ Therefore, L-RB is expected to be very useful as an ion-association reagent for the sensing membrane of an optical sensor for the determination of anionic surfactants.

In this work, we prepared and characterized an optical sensing membrane based on L-RB in order to evaluate its potential use for the detection of anionic surfactants.

\section{Experimental}

\section{Chemicals}

Sodium dodecylsulfate (NaDS) and sodium dodecylbenzenesulfonate (NaDBS) of guaranteed grade were obtained from Wako Pure Chemicals Co. Sodium di-2-ethylhexyl sulfosuccinate (NaSS) (purity, 96.3\%) was obtained from Kanto Chemicals Co. PVC (degree of polymerization, 1100) and RB were obtained from Wako Pure Chemicals Co. 2-Nitrophenyloctyl ether (NPOE) and tetradecyldimethylbenzylammonium chloride (Zephiramine) were obtained from Dojindo Laboratories. All other chemicals were of guaranteed grade and used as received.

Preparation of an anionic surfactant-selective optical PVC membrane based on $L-R B$

An anionic surfactant-selective optical plasticized PVC membrane containing L-RB as an ion-association reagent was prepared as follows. A $4 \mathrm{~mL}$ aqueous solution containing $2.5 \times$ $10^{-3} \mathrm{M} \mathrm{RB}$ and $1.0 \mathrm{M} \mathrm{NaOH}$, and NPOE $(1.0 \mathrm{~g})$ were mixed in a test tube and stirred for $24 \mathrm{~h}$ at $50^{\circ} \mathrm{C}$ in order to extract L-RB in the NPOE phase. After the NPOE phase and the water phase were separated by centrifugation (10 min), the NPOE phase and PVC powder $(0.40 \mathrm{~g})$ were dissolved in $10 \mathrm{~mL}$ of tetrahydrofuran (THF). A glass plate (length, $3.5 \mathrm{~cm}$; width, 1.0 $\mathrm{cm}$; thickness, $1 \mathrm{~mm}$ ) was coated with the optical PVC membrane by dipping it in the THF solution for $5 \mathrm{~s}$. The glass plate was dried for more than $2 \mathrm{~h}$ at room temperature.

\section{Standard measurement procedure}

Glass plates coated with optical PVC membranes were fixed in disposable cuvettes $(4.5 \mathrm{~cm}$ high, $1.25 \mathrm{~cm}$ long, and $1.25 \mathrm{~cm}$ wide) filled with $2.0 \mathrm{~mL}$ of an anionic surfactant solution $(\mathrm{pH}$ 4.0 , adjusted with a $0.1 \mathrm{M} \mathrm{CH} \mathrm{CH}_{3} \mathrm{COOH} / \mathrm{CH}_{3} \mathrm{COONa}$ buffer solution). The anionic surfactant solution contained a $10.0 \%(\mathrm{v} / \mathrm{v})$ buffer solution. The absorption spectrum of the membrane was measured with a spectrophotometer (JASCO, V-530-iRM). All spectrometric measurements were performed in a batch mode. The membranes were used for one-shot measurements (as a disposable sensor).

\section{Results and Discussion}

Absorption spectra of an optical sensing membrane after exposure to a NaDBS solution

Figure 1 shows the absorption spectra of an optical PVC membrane after exposure to a $50 \mu \mathrm{M}$ NaDBS solution ( $\mathrm{pH} 4.0$, adjusted with a $0.1 \mathrm{M} \mathrm{CH} \mathrm{CHOOH}_{3} \mathrm{CH} \mathrm{H}_{3} \mathrm{COONa}$ buffer solution) and a $\mathrm{pH} 4.0$ buffer solution for $30 \mathrm{~min}$, respectively. After exposure to a $50 \mu \mathrm{M}$ NaDBS solution for $30 \mathrm{~min}$, the absorption band of L-RB at $558 \mathrm{~nm}$ (absorption maximum) was observed, although the absorption band of L-RB at $558 \mathrm{~nm}$ was almost not observed after contact with the $\mathrm{pH} 4.0$ buffer solution for 30 min. The absorption band of L-RB at $558 \mathrm{~nm}$ for the membrane exposed to the $50 \mu \mathrm{M}$ NaDBS solution was apparent due to

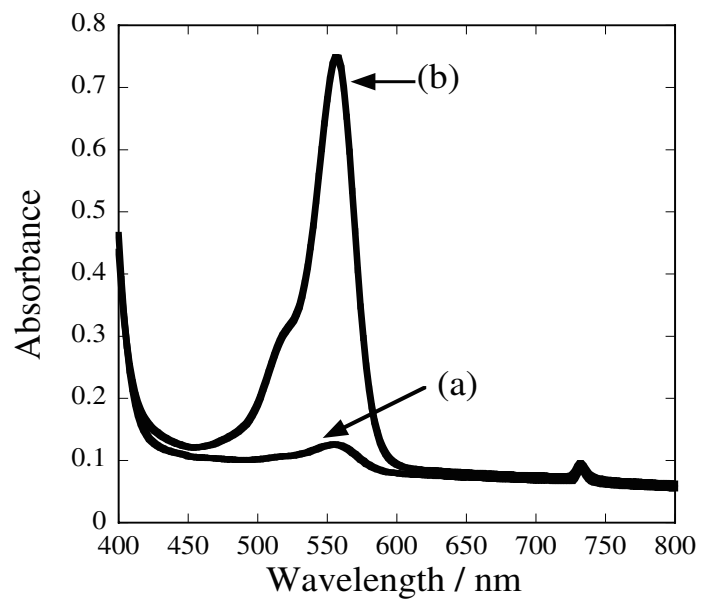

Fig. 1 Absorption spectra of an optical PVC membrane after exposure to a $50 \mu \mathrm{M}$ NaDBS solution $(\mathrm{pH} 4.0$, adjusted with a $0.1 \mathrm{M}$ $\mathrm{CH}_{3} \mathrm{COOH} / \mathrm{CH}_{3} \mathrm{COONa}$ buffer solution) and a $\mathrm{pH} 4.0$ buffer solution, respectively. (a) After exposure to a $\mathrm{pH} 4.0$ buffer solution for 30 min. (b) After exposure to a $50 \mu \mathrm{M}$ NaDBS solution for $30 \mathrm{~min}$.

ion-pair coextraction of the $\mathrm{DBS}^{-}$ion and a proton into the membrane. In this case, the color of the membrane changed from light pink to dark pink upon exposure to the NaDBS solution. Namely, the L-RB protonates formed an ion associate with the extracted anionic surfactant; simultaneously, the protonation of the L-RB was accompanied by a spectral change. The ion-pair coextraction reaction was as follows:

$$
\begin{array}{r}
\mathrm{AS}^{-}{ }_{\mathrm{aq}}+\mathrm{H}^{+}{ }_{\mathrm{aq}}+(\mathrm{L}-\mathrm{RB})_{\mathrm{memb}}(\text { light pink }) \rightleftharpoons \\
\left(\mathrm{L}-\mathrm{RB}-\mathrm{H}^{+}-\mathrm{AS}^{-}\right)_{\mathrm{memb}}(\text { dark pink }),
\end{array}
$$

where $\mathrm{AS}^{-}$is an anionic surfactant, memb is an optical membrane, and aq is an aqueous solution.

Next, the effect of the $\mathrm{pH}$ in the sample solution on the sensitivity of the optical membrane to the $50 \mu \mathrm{M}$ NaDBS solution was examined. Figure 2 shows the effect of the $\mathrm{pH}$ in the sample solution on the absorption spectra of the optical membrane after exposure to $50 \mu \mathrm{M}$ NaDBS solutions and several $\mathrm{pH}$ buffer solutions for $30 \mathrm{~min}$, respectively. The absorbance of the PVC membrane at $558 \mathrm{~nm}$ for $50 \mu \mathrm{M}$ NaDBS solutions showed the maximum value at $\mathrm{pH} 2.2$ among several $\mathrm{pH}$ buffer solutions. This means that the $\mathrm{AS}^{-}$ion was most extracted into the PVC membrane at $\mathrm{pH}$ 2.2. The result is in accord with the result of FIA of anionic surfactants coupled with liquid-liquid extraction using L-RB reported by Motomizu and Kobayashi. ${ }^{17}$ They used a sample solution containing $0.05 \mathrm{M}$ $\mathrm{H}_{2} \mathrm{SO}_{4}$. However, the absorbance of the PVC membrane at 588 $\mathrm{nm}$ for buffer solutions containing no NaDBS also showed the maximum value at $\mathrm{pH} 2.2$ among several buffer solutions. From Figs. 1 and 2, the difference between the absorbance of the PVC membrane for $50 \mu \mathrm{M}$ NaDBS solutions containing several buffer solutions and that for the same buffer solutions shows the maximum value at $\mathrm{pH} 4.0$. From these results, $\mathrm{pH} 4.0$ was chosen for measuring the absorbance of the optical PVC membrane.

\section{Response time and calibration curve for anionic surfactants}

Figure 3 shows typical dynamic-response curves of the optical PVC membrane recorded at $558 \mathrm{~nm}$ as a result of exposure to a $1-50 \mu \mathrm{M}$ NaDBS solution for $30 \mathrm{~min}$. The absorbance of the PVC membrane increases as the exposure time increases. This may be because the extraction rate of anionic surfactants, such 

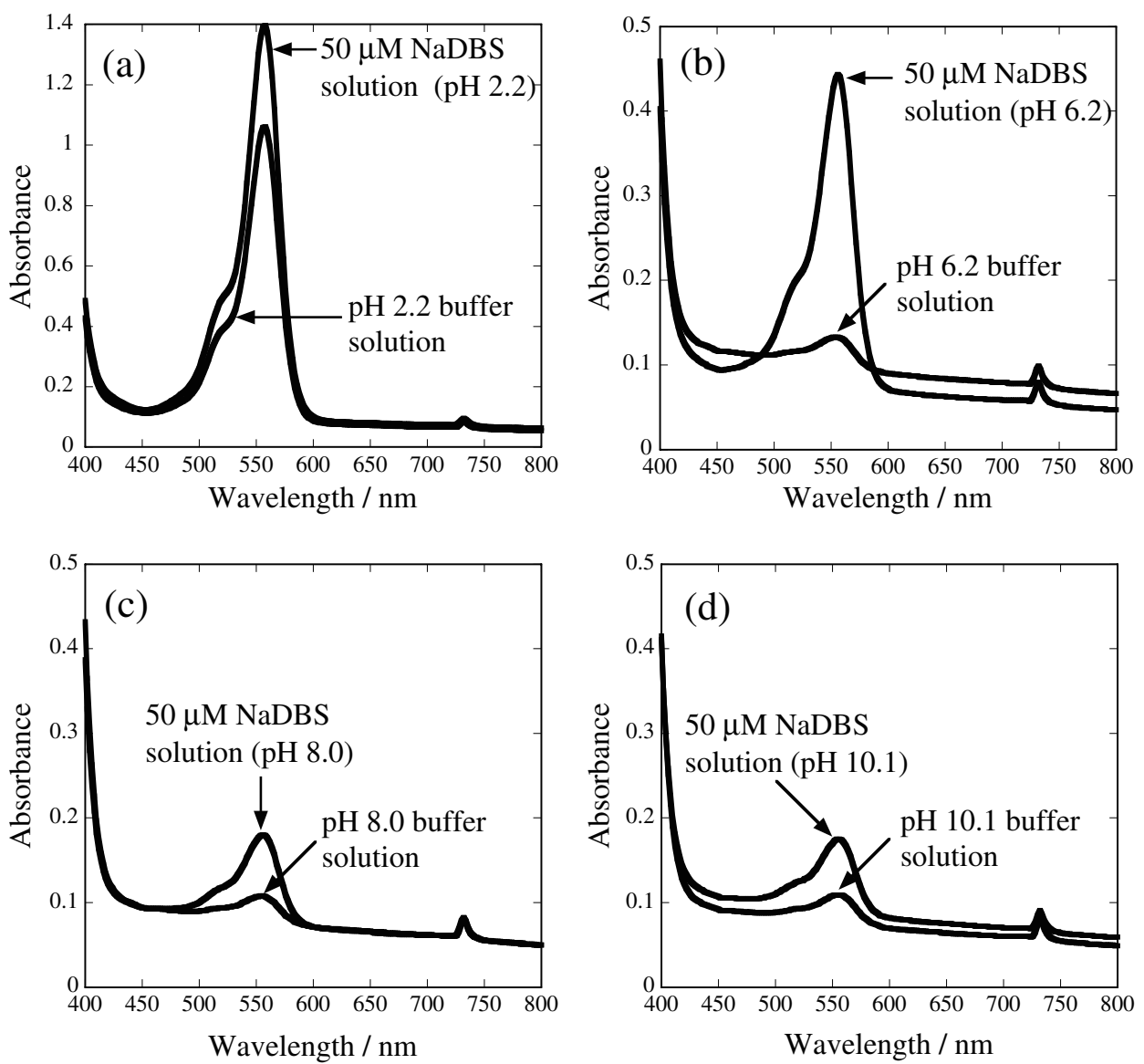

Fig. 2 Effect of the $\mathrm{pH}$ in the sample solution on the absorption spectra of the optical membrane after exposure to $50 \mu \mathrm{M}$ NaDBS solutions and several $\mathrm{pH}$ buffer solutions for $30 \mathrm{~min}$, respectively. $\mathrm{pH}$ in the sample solution: (a) 2.2, (b) 6.2, (c) 8.0, (e) 10.1 .

as NaDBS in the optical membrane is very slow, and hence a very long response time is obtained. However, in the case of an exposure time of $10 \mathrm{~min}$, the PVC membrane responds to NaDBS in the concentration range from 1 to $50 \mu \mathrm{M}$. An exposure time of 10 min was used in subsequent experiments as a compromise in the sensitivity of the optical membrane and the analysis time.

At an exposure time of 10 min, a linear relationship was obtained between the absorbance of the optical membrane and the concentration of NaDBS in the concentration range from 1 to $50 \mu \mathrm{M}$. The dynamic-response curve of the optical PVC membrane to several anionic surfactant solutions other than the NaDBS solution is similar to that to the NaDBS solution. The calibration curves at an exposure time of $10 \mathrm{~min}$ are also identical for anionic surfactants other than NaDBS, i.e., NaDS and NaSS. This means that the optical PVC membrane determines the total anionic surfactants in commercial products, such as detergents. The detection limit was $c a .1 \mu \mathrm{M}$ for these anionic surfactants. The reproducibility of the absorbance of an optical PVC membrane exposed to NaDBS solutions was examined using three different membranes. The relative standard deviations of the absorbance of the membranes were found to be $c a$. $1.6 \%$ for $5 \mu \mathrm{M}, c a .3 .2 \%$ for $20 \mu \mathrm{M}, c a .0 .63 \%$ for $40 \mu \mathrm{M}$, and $c a .2 .9 \%$ for $50 \mu \mathrm{M}$, respectively.

Effect of coexisting species on the determination of anionic surfactants

Real samples, such as environmental water, commercial

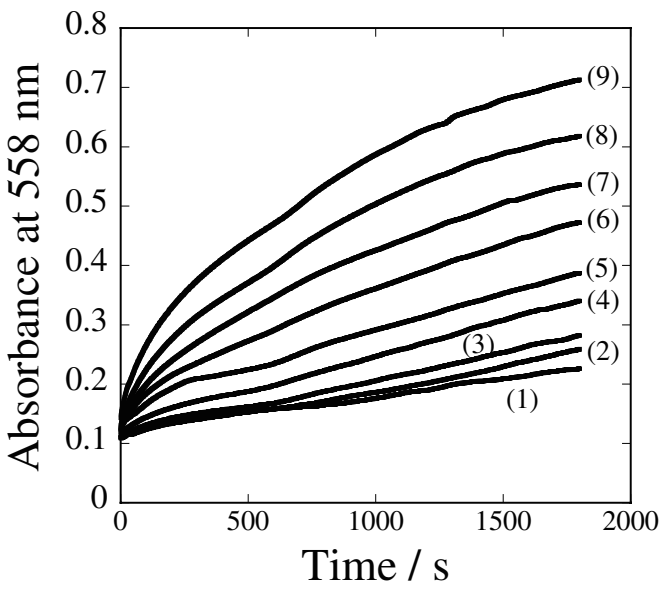

Fig. 3 Typical dynamic-response curves for an optical PVC membrane recorded at $558 \mathrm{~nm}$ as a result of exposure to NaDBS solutions ( $\mathrm{pH} 4.0$, adjusted with $0.1 \mathrm{M} \mathrm{CH}_{3} \mathrm{COOH} / \mathrm{CH}_{3} \mathrm{COONa}$ buffer solution). Concentration of NaDBS: (1) 0 , (2) 1, (3) 2, (4) 5, (5) 10 , (6) 20, , (7) 30, (8) 40 , (9) $50 \mu \mathrm{M}$.

detergents and emulsifying agents, often contain common cations, anions and nonionic surfactants. Therefore, the effect of these coexisting species on the determination of anionic surfactants was examined in order to apply the present method 
to the determination of anionic surfactants in real samples. The effect of common inorganic cations and anions on the determination of $30 \mu \mathrm{M}$ NaDBS was examined. The coexistence of common cations and anions, such as $\mathrm{Na}^{+}, \mathrm{K}^{+}, \mathrm{Cl}^{-}$, $\mathrm{Ca}^{2+}$ and $\mathrm{Mg}^{2+}$ ions at 1000 -times excess to $\mathrm{NaDBS}$, had no effect on the absorbance of a PVC membrane exposed to $30 \mu \mathrm{M}$ NaDBS within an error of $\pm c a$. $5 \%$. However, the coexistence of the $\mathrm{NO}_{3}{ }^{-}$ion at 1000 -times excess to $\mathrm{NaDBS}$ gave a positive error of $c a .10 \%$ on the absorbance of a PVC membrane exposed to $30 \mu \mathrm{M}$ NaDBS. The effect of a nonionic surfactant (Triton X-100) on the determination of $30 \mu \mathrm{M}$ NaDBS was examined. The coexistence of Triton X-100 at 5-times excess to NaDBS had no effect on the absorbance of the PVC membrane exposed to $30 \mu \mathrm{M}$ NaDBS. The effect of a cationic surfactant (Zephiramine) on the determination of $30 \mu \mathrm{M} \mathrm{NaDBS}$ was also examined. The existence of $15 \mu \mathrm{M}$ Zephiramine on the determination of $30 \mu \mathrm{M}$ NaDBS gave a serious negative error of ca. 23\%. Therefore, coexisting cationic surfactants in the sample solution should be removed before the determination of an anionic surfactant with the present optical sensor for an anionic surfactant.

\section{Conclusions}

Anionic surfactants are determined by an optical PVC membrane incorporating L-RB as an optical sensing reagent in a concentration range from 1 to $50 \mu \mathrm{M}$. The proposed optical membrane method is very simple to perform, and the fabrication of an optical membrane is very easy. The sensitivity of the optical PVC membrane is much higher than that of other optical sensors. ${ }^{15}$ Furthermore, L-RB as an optical sensing reagent for the determination of anionic surfactants is much cheaper than other optical sensing reagents, and is easily obtained by a solvent-extraction method. The method is simpler than the conventional solvent extraction-spectrophotometric methods, and no poisonous organic solvent is used. The ion-pair coextraction reaction (1) of anionic surfactants and a proton into the membrane is expected to be reversible. Indeed, the optical PVC membrane can be used as a sensor with a reversible response by using a $\mathrm{NaOH}$ solution as a regeneration solution. However, L-RB in the membrane is often leached into the sample solution by using a $\mathrm{NaOH}$ solution as a regeneration solution; this may be due to the low hydrophobicity of L-RB. Therefore, the optode membrane should only be used as a oneshot membrane at this stage.

The present method will be very useful for the quality control of detergent manufacturing and for the determination of anionic surfactants in environmental water. Detailed results, including a comparison of the present method with a popular current method for the determination of anionic surfactants in real samples, will be reported elsewhere.

\section{References}

1. Japanese Industrial Standard Committee, "JIS-K 0120", 1986, Japanese Standards Association, Tokyo.

2. S. Motomizu, S. Fujiwara, A. Fujiwara, and K. Toei, Anal. Chem., 1982, 54, 392.

3. S. Taguchi, I. Kasahara, Y. Fukushima, and K. Goto, Talanta, 1981, 28, 616.

4. H. Hisamoto, Y. Manabe, H. Yanai, H. Tohma, T. Yamada, and K. Suzuki, Anal. Chem., 1998, 70, 1255.

5. H. Hisamoto, S. Sato, K. Sato, D. Siswanta, and K. Suzuki, Anal. Sci., 1998, 14, 127.

6. W. H. Chan, A. W. M. Lee, J. Lu, and X. Wu, Anal. Chim. Acta, 1998, 370, 259.

7. M. M. F. Choi, X. J. Wu, and Y. R. Li, Anal. Chem., 1999, $71,1342$.

8. C. Sanchez-Pedreno, J. A. Ortuno, M. I. Albero, M. S. Garcia, and J. C. Garcia de las Bayonas, Fresenius' J. Anal. Chem., 2000, 366, 811.

9. I. M. Steinberg, A. Lobnik, and O. S. Wolfbeis, Sens. Actuators, B, 2003, 81, 230.

10. T. Masadome and T. Takahashi, Anal. Lett., 2007, 40, 441.

11. W. R. Seitz and Z. M. Shakhsher, Anal. Chem., 1990, 62, 1758.

12. J. S. Lundgren and F. V. Bright, Anal. Chem., 1996, 68, 3377.

13. Y. Kawabata, T. Yamamoto, and T. Imasaka, Sens. Actuators, B, 1993, 11, 341.

14. E. Wang, H. Chen, H. Patel, I. Sadaragani, and C. Romero, Anal. Chim. Acta, 1999, 397, 287.

15. W. H. Chan, A. W. M. Lee, and J.-Z. Lu, Anal. Chim. Acta, 1998, 361, 55.

16. D. Yamamoto and T. Abe, Meiji Daigaku Nogakubu Kenkyu Hokoku, 1988, 78, 31.

17. S. Motomizu and M. Kobayashi, Anal. Chim. Acta, 1992, 261, 471.

18. C. Preininger, G. J. Mohr, I. Klimanta, and O. S. Wolfbeis, Anal. Chim. Acta, 1996, 334, 113.

19. C. Preininger and G. J. Mohr, Anal. Chim. Acta, 1997, 342, 207.

20. A. A. El-Rayyes, A. A.-Betar, T. Htun, and U. K. A. Klein, Chem. Phys. Lett., 2005, 414, 287.

21. A. Silva, R. E. F. Boto, R. M. El-Shishtawy, and P. Almeida, Eur. Polym. J., 2006, 42, 2270. 